\title{
SYNCHRONIZATION IN THE SECOND APPROXIMATION
}

\author{
NGUYEN VAN DINH \\ Institute of Mechanics
}

It is well-known the phenomenon of synchronization (frequency entrainment) in self-excited systems subjected to external or parametric excitations; A lot of monographs $[2,3,4]$ has analyzed several important systems for which the synchronization effect occurs and can be determined in the first approximation. However, there exist also certain systems possessing a stable-excited oscillation obtained in the first approximation, which may be synchronized only in the second approximation. This article deals with some systems of the mentioned type; the asymptotic method [1] is applied for this purpose.

1. System under consideration and the solution in the second approximation

Let us consider the system governed by the differential equation of the form:

$$
\ddot{x}+x=\varepsilon f(x, \dot{x}, n \omega t)=\varepsilon\left\{h\left(1-x^{2}\right) \dot{x}+g(x, n \omega t)\right\},
$$

where $x$ is an oscillatory variable; overdots denote differentiation with respect to time $t ; 1$ is the own frequency; $\varepsilon>0$ is a small formal parameter; $h>0$ is the intensity of the self-excitation $h\left(1-x^{2}\right) \dot{x} ; g(x, n \omega t)$ represents external or parametric excitations of frequency $n \omega$ ( $g$ will be given below for each case examined).

Assuming that $\omega$ is very close to the own frequency (pratically, the system is in exact resonance) we rewrite (1.1) as:

$$
\ddot{x}+\omega^{2} x=\varepsilon f(x, \dot{x}, n \omega t)+\varepsilon^{2} \Delta x,
$$

where $\varepsilon^{2} \Delta=\omega^{2}-1$ is the detuning parameter of order $\varepsilon^{2}$.

According to the asymptotic method, following expansions are used:

$$
\begin{aligned}
& x=a \cos \psi+\varepsilon u_{1}(a, \theta, \psi)+\varepsilon^{2} u_{2}(a, \theta, \psi)+\ldots, \quad \psi=\omega t+\theta, \\
& \dot{a}=\varepsilon A_{1}(a, \theta)+\varepsilon^{2} A_{2}(a, \theta)+\ldots, \\
& \dot{\theta}=\varepsilon B_{1}(a, \theta)+\varepsilon^{2} B_{2}(a, \theta)+\ldots,
\end{aligned}
$$

where $a, \theta$ are full amplitude and dephase of the first harmonic; $A_{i}, B_{i}(i=1,2, \ldots)$ are functions of $a, \theta$; and $u_{i}(i=1,2, \ldots)$ are functions of $a, \theta, \psi, 2 \pi$-periodic 
with respect to $\psi$ and do not contain first harmonics $\cos \psi, \sin \psi$. With regard to (1.4), (1.5), substituting (1.3) into (1.2), expanding $f(x, \dot{x}, n \omega t)$ in Taylor serie of $\varepsilon$, equating the terms of like powers of $\varepsilon$ yield.

In the first approximation:

$$
-2 \omega A_{1} \sin \psi-2 \omega a B_{1} \cos \psi+\omega^{2}\left(\frac{\partial^{2} u_{1}}{\partial \psi^{2}}+u_{1}\right)=f\left(a \cos \psi_{1}-\omega a \sin \psi_{1} n \psi-n \theta\right) .
$$

In the second approximation

$$
\begin{aligned}
- & 2 \omega A_{2} \sin \psi-2 \omega a B_{2} \cos \psi_{1}+\omega^{2}\left(\frac{\partial^{2} u_{2}}{\partial \psi^{2}}+u_{2}\right)= \\
= & -A-1 \frac{\partial A_{1}}{\partial a} \cos \psi-2 \omega A_{1} \frac{\partial^{2} u_{1}}{\partial \psi \partial a}+u_{i} f_{x}\left(a \cos \psi_{1}-\omega a \sin \psi_{1} n \psi-n \theta\right) \\
& +\left(A_{1} \cos \psi+\omega \frac{\partial u_{1}}{\partial \psi}\right) f_{\dot{x}}\left(a \cos \psi_{1}-\omega a \sin \psi_{1} n \psi-n \theta\right)+\delta a \cos \psi+\ldots,
\end{aligned}
$$

where $f_{x}, f_{\dot{x}}$ are partial derivatives with respect to $x, \dot{x}$ and non-written terms contain $B_{1}$ as a factor. $A_{1}, B_{1}, u_{1}$ and $A_{2}, B_{2}, u_{2}$ are obtained by equating the terms of like harmonics in both sides of (1.6), (1.7). If $A_{1}$ does not contain $\theta$ and $B_{1} \equiv 0$, the amplitude and the dephase of synchronized oscillations (if it exists) are determined by the equations:

$$
\begin{aligned}
\varepsilon A_{1}(a)+\varepsilon^{2} A_{2}(a, \theta) & =0 \\
\varepsilon^{2} B_{2}(a, \theta) & =0 .
\end{aligned}
$$

2. Synchronization under external excitation in subharmonic resonance of order $1 / 3$

First, consider the case of an external excitation of intensity $e>0$ and frequency $3 \omega$, that is

$$
g(x, n \omega t)=e \cos 3 \omega t .
$$

In the first approximation we have

$$
\begin{aligned}
- & 2 \omega A_{1} \sin \psi-2 \omega a B_{1} \cos \psi+\omega^{2}\left(\frac{\partial^{2} u_{1}}{\partial \psi^{2}}+u_{1}\right)= \\
= & -h \omega a\left(1-\frac{a^{2}}{4}\right) \sin \psi+\frac{h \omega a^{3}}{4} \sin 3 \psi+e \cos (3 \psi-3 \theta), \\
A_{1}= & \frac{h a}{2}\left(1-\frac{a^{2}}{4}\right), \quad B_{1}=0, \\
u_{1}= & -\frac{h \omega a^{2}}{32 \omega^{2}} \sin 3 \psi-\frac{e}{8 \omega^{2}} \cos (3 \psi-3 \theta) .
\end{aligned}
$$


Hence, the expansions (1.4), (1.5) are:

$$
\dot{a}=\frac{\varepsilon h a}{2}\left(1-\frac{a^{2}}{4}\right), \quad \dot{\theta}=0 .
$$

Evidently, in the first approximation, there exists only a stationary self-excited oscillation with amplitude $a_{*}=2$ and arbitrary (indetermined) dephase $\theta$; this oscillation is stable (in amplitude) since $\frac{\partial A_{1}\left(a_{*}\right)}{\partial a}=-h<0$, it is perturbed by small oscillations (2.5); the latter are due to the'external excitation and also to the non-linear character of the self-excitation. The synchronization cannot be revealed by the calculation in the first approximation.

In the second approximation (with regard that $B_{1}=0$ )

$$
\begin{aligned}
&- 2 \omega A_{2} \sin \psi-2 \omega a B_{2} \cos \psi+\omega^{2}\left(\frac{\partial^{2} u_{2}}{\partial \psi^{2}}+u_{2}=-A_{1} \frac{\partial A_{1}}{\partial a} \cos \psi-2 \omega A_{1} \frac{\partial^{2} u_{1}}{\partial \psi \partial a}\right. \\
&+u_{1} \cdot h \omega a^{2} \sin 2 \psi+\left(A_{1} \cos \psi+\omega \frac{\partial u_{1}}{\partial \psi}\right) h\left(1-a^{2} \cos ^{2} \psi\right)+\Delta a \cos \psi, \\
& A_{2}= \frac{h e a^{2}}{64 \omega^{2}} \cos 3 \theta \\
& B_{2}=\frac{-1}{2 \omega a}\left\{\frac{h \omega e a^{2}}{32 \omega^{2}} \sin 3 \theta+\frac{h^{2} \omega^{2} a^{5}}{128 \omega^{2}}+\left(\omega^{2}-1\right) a-A_{1} \frac{\partial A_{1}}{\partial a}+h A_{1}\left(1-\frac{3 a^{2}}{4}\right)\right\} .
\end{aligned}
$$

By $a_{s}, \theta_{s}$ we denote the amplitude and dephase of synchronized oscillations; they are determined (as it has been noted above) by the equations (1.8), (1.9).

By letting

$$
a_{s}=a_{*}+\varepsilon a_{1}=2+\varepsilon a_{1},
$$

and expanding $A_{1}\left(a_{*}+\varepsilon a_{1}\right)$ in Taylor serie of $\varepsilon$, the equation (1.8) becomes

$$
\varepsilon A_{1}\left(a_{*}\right)+\varepsilon^{2} a_{1} \frac{\partial A_{1}\left(a_{\alpha}\right)}{\partial a}+\varepsilon^{2} A_{2}\left(a_{*}, \theta_{s}\right)=0 .
$$

With regard that $A_{1}\left(a_{*}\right)=A_{1}(2)=0$, from (2.10), one obtains:

$$
a_{1}=-A_{2}\left(a_{*}, \theta_{s}\right) / \frac{\partial A_{1}\left(a_{*}\right)}{\partial a}=\frac{e \cos 3 \theta_{s}}{16 \omega^{2}} .
$$

The dephase $\theta_{s}$ is given by the equation (1.9), that is:

$$
\frac{h \omega e a_{*}^{2}}{32 \omega^{2}} \sin 3 \theta_{s}+\frac{h^{2} \omega^{2} a_{*}^{5}}{128 \omega^{2}}+\left(\omega^{2}-1\right) a_{*}=0
$$

or

$$
\sin 3 \theta_{s}=\frac{-2 \omega}{h e}\left[h^{2}+8\left(\omega^{2}-1\right)\right]
$$


with the condition that

$$
-h e \leq 2 \omega\left[h^{2}+8\left(\omega^{2}-1\right)\right] \leq h e .
$$

If $\omega^{2}=1$ (exact resonance), $\sin 3 \theta_{s}=-\frac{2 h}{e}$ with the condition that $e \geq 2 h, a_{1}=$ $\pm \frac{e}{16} \sqrt{1-\frac{4 h^{2}}{e^{2}}}, a_{s}=2 \pm \frac{e}{16} \sqrt{1-\frac{4 h^{2}}{e^{2}}}$. Note that there exist two amplitudes: the larger corresponds to $\cos 3 \theta_{s}>0$ i.e. $a_{1}>0$, the smaller corresponds to $\cos 3 \theta_{s}<0$ i.e. $a_{1}<0$.

3. Synchronization under linear parametric excitation in fundamental resonance

The second case to be examined is that of a linear parametric excitation of intensity $2 p>0$ and frequency $\omega$ i.e.

$$
g(x, n \omega t)=2 p x \cos \omega t .
$$

In the first approximation:

$$
\begin{aligned}
- & 2 \omega A_{1} \sin \psi-2 \omega a B_{1} \cos \psi+\omega^{2}\left(\frac{\partial^{2} u_{1}}{\partial \psi_{2}}+u_{1}\right)=-h \omega a\left(1-\frac{a^{2}}{4}\right) \sin \psi \\
& +\frac{h \omega a^{3}}{4} \sin 3 \psi+p a \cos \theta+p a \cos (2 \psi-\theta) \\
A_{1}= & \frac{h a}{2}\left(1-\frac{a^{2}}{4}\right), \quad B_{1}=0 \\
u_{1}= & -\frac{h \omega a^{3}}{32 \omega^{2}} \sin 3 \psi+\frac{1}{\omega^{2}} p a \cos \theta-\frac{1}{3 \omega^{2}} p a \cos (2 \psi-\theta) .
\end{aligned}
$$

In the second approximation

$$
\begin{aligned}
- & 2 \omega A_{2} \sin \psi-2 \omega a B_{2} \cos \psi+\omega^{2}\left(\frac{\partial^{2} u_{2}}{\partial \psi^{2}}+u_{2}\right) \\
= & -A_{1} \frac{\partial A_{1}}{\partial a} \cos \psi-2 \omega A_{1} \frac{\partial^{2} u_{1}}{\partial \psi \partial a}+u_{1}\left\{h \omega a^{2} \sin 2 \psi+2 p \cos (\psi-\theta)\right\} \\
& +\left(A_{1} \cos \psi+\omega \frac{\partial u_{1}}{\partial \psi}\right) h\left(1-a^{2} \cos ^{2} \psi\right)+\delta a \cos \psi \\
A_{2}= & -\frac{p^{2} a \sin 2 \theta}{4 \omega^{3}} \\
B_{2}= & \frac{-1}{2 \omega a}\left\{\frac{p^{2} a}{\omega^{2}} \cos ^{2} \theta-\frac{p^{2} a}{3 \omega^{2}}+\frac{h^{2} \omega^{2} a^{5}}{128 \omega^{2}}+\left(\omega^{2}-1\right) a-A_{1} \frac{\partial A_{1}}{\partial a}+h A_{1}\left(1-\frac{3 a^{2}}{4}\right\}\right. \\
\cos ^{2} \theta_{s}= & \frac{1}{3}-\frac{h^{2} \omega^{2}}{8 p^{2}}-\frac{\omega^{2}\left(\omega^{2}-1\right)}{p^{2}}, \quad a_{1}=-\frac{p^{2} \sin 2 \theta_{s}}{4 h \omega^{3}}, \quad a_{s}=2-\frac{p^{2} \sin 2 \theta_{s}}{4 h \omega^{3}}
\end{aligned}
$$


If $\omega^{2}=1, a_{s}=2-\frac{p^{2} \sin 2 \theta_{s}}{4 h}, \cos ^{2} \theta_{s}=\frac{1}{3}-\frac{h^{2}}{8 p^{2}}$ on condition that $p^{2} \geq \frac{3}{8} h^{2}$.

4. Synchronization under quadratic parametric excitation in subharmonic resonance of order $\frac{1}{2}$

For the third example, consider the case

$$
g(x, n \omega t)=2 p x^{2} \cos 2 \omega t,
$$

where $2 p>0$ and $2 \omega$ are intensity and frequency of a quadratic parametric excitation in subharmonic resonance of order $\frac{1}{2}$.

In the first approximation

$-2 \omega A_{1} \sin \psi-2 \omega a B_{1} \cos \psi+\omega^{2}\left(\frac{\partial^{2} u_{1}}{\partial \psi^{2}}+u_{1}\right)=-h \omega a\left(1-\frac{a^{2}}{4}\right) \sin \psi$

$$
+\frac{h \omega a^{3}}{4} \sin 3 \psi+\frac{1}{2} p a^{2} \cos 2 \theta+p a^{2} \cos (2 \psi-2 \theta)+\frac{1}{2} p a^{2} \cos (4 \psi-2 \theta),
$$

$A_{1}=\frac{h a}{2}\left(1-\frac{a^{2}}{4}\right), \quad B_{1}=0$,

$u_{1}=-\frac{h \omega a^{3}}{32 \omega^{2}} \sin 3 \psi+\frac{1}{2 \omega^{2}} p a^{2} \cos 2 \theta-\frac{p a^{2}}{3 \omega^{2}} \cos (2 \psi-2 \theta)-\frac{1}{30 \omega^{2}}\left(2 a^{2} \cos (4 \psi-2 \theta)\right.$.

In the second approximation

$-2 \omega A_{2} \sin \psi-2 \omega a B_{2} \cos \psi+\omega^{2}\left(\frac{\partial^{2} u_{2}}{\partial \psi^{2}}+u_{2}\right)=-A_{1} \frac{\partial A_{1}}{\partial a} \cos \psi-2 \omega A_{1} \frac{\partial^{2} u_{1}}{\partial \psi \partial a}$

$$
+u_{2}\left\{h \omega a^{2} \sin 2 \psi+4 p a \cos \psi \cos (2 \psi-2 \theta)\right\}
$$$$
+\left(A_{1} \cos \psi+\omega \frac{\partial u_{1}}{\partial \psi}\right) h\left(1-A^{2} \cos ^{2} \psi\right)+\Delta a \cos \psi,
$$

$A_{2}=\frac{-1}{8 \omega^{3}} p^{2} a^{3} \sin ^{4} \theta$

$B_{2}=\frac{-1}{2 \omega a}\left\{\frac{p^{2} a^{3}}{2 \omega^{2}} \cos ^{2} 2 \theta-\frac{21 p^{2} a^{3}}{30 \omega^{2}}+\frac{h^{2} \omega^{2} a^{5}}{128 \omega^{2}}\right.$

$$
\left.+\left(\omega^{2}-1\right) a-A_{1} \frac{\partial A_{1}}{\partial a}+h A_{1}\left(1-\frac{3 a^{2}}{4}\right)\right\}
$$

$a_{1}=-\frac{p^{2} \sin 4 \theta_{s}}{h \omega^{3}}, a_{s}=2-\frac{p^{2} \sin 4 \theta_{s}}{h \omega^{3}}, \cos ^{2} 2 \theta_{s}=\frac{7}{5}-\frac{h^{2} \omega^{2}}{16 p^{2}}-\frac{\omega^{2}\left(\omega^{2}-1\right)}{2 p^{2}}$.

If $\omega^{2}=1, a_{s}=2-\frac{p^{2} \sin 4 \theta_{s}}{h}, \cos ^{2} 2 \theta_{s}=\frac{7}{5}-\frac{h^{2}}{16 p^{2}}$ with the condition that

$$
\frac{5 h^{2}}{112} \leq p^{2} \leq \frac{5 h^{2}}{32} \text {. }
$$


5. Synchronization under the interaction between quadratic nonlinearity and excitation in subharmonic resonance of order $\frac{1}{2}$

The last example is devoted to the case in which $g(x, n \omega t)$ consists of the quadratic nonlinearity $-\beta x^{2}(\beta>0$ and the external excitation $e \cos 2 \omega t$

$$
g(x, n \omega t)=-\beta x^{2}+e \cos 2 \omega t .
$$

If $\beta=0$, the self-excited oscillation cannot be synchronized; if $\beta>0$, under certain condition, the synchronization may occur in the second approximation.

In the first approximation

$$
\begin{aligned}
& -2 \omega A_{1} \sin \psi-2 \omega a B_{1} \cos \psi+\omega^{2}\left(\frac{\partial^{2} u_{1}}{\partial \psi^{2}}+u_{1}\right)=-h \omega a\left(1-\frac{a^{2}}{4} \sin \psi\right) \\
& \quad+\frac{h \omega a^{3}}{4} \sin 3 \psi-\beta a^{2} \cos ^{2} \psi+e \cos (2 \psi-2 \theta), \\
& A_{1}=\frac{h a}{2}\left(1-\frac{a^{2}}{4}\right), \quad B_{1}=0, \\
& u_{1}=-\frac{h \omega a^{3}}{32 \omega^{2}} \sin 3 \psi-\frac{\beta a^{2}}{2 \omega^{2}}+\frac{\beta a^{2}}{6 \omega^{2}} \cos 2 \psi-\frac{e}{3 \omega^{2}} \cos (2 \psi-2 \theta) .
\end{aligned}
$$

In the second approximation:

$$
\begin{aligned}
& -2 \omega A_{2} \sin \psi-2 \omega a B_{2} \cos \psi+\omega^{2}\left(\frac{\partial^{2} u_{2}}{\partial \psi^{2}}+u_{2}\right)=-A_{1} \frac{\partial A_{1}}{\partial a} \cos \psi-2 \omega A_{1} \frac{\partial^{2} u_{1}}{\partial \psi \partial a} \\
& +u_{1}\left\{h \omega a^{2} \sin 2 \psi-2 \beta a \cos \psi\right\}+\left(A_{1} \cos \psi+\omega \frac{\partial u_{1}}{\partial \psi}\right) h\left(1-a^{2} \cos ^{2} \psi\right) \\
& +\Delta a \cos \psi,
\end{aligned}
$$

$A_{2}=-\frac{\beta a e}{6 \omega^{3}} \sin 2 \theta$

$B_{2}=-\frac{1}{2 \omega a}\left\{\frac{\beta a e}{2 \omega^{2}} \cos 2 \theta+\frac{\beta^{2} a^{3}}{3 \omega^{2}}+\frac{h^{2} \omega^{2} a^{5}}{128 \omega^{2}}+\left(\omega^{2}-1\right) a-A_{1} \frac{\partial A_{1}}{\partial a}+h A_{1}\left(1-\frac{3 a^{2}}{4}\right)\right\}$,

$a_{1}=-\frac{\beta e}{3 h \omega^{3}} \sin 2 \theta_{s}, a_{s}=2-\frac{\beta e}{3 h \omega^{3}} \sin 2 \theta_{s}, \cos 2 \theta_{s}=-\frac{4 \beta}{e}-\frac{3 h^{2} \omega^{2}}{8 \beta e}-\frac{3 \omega^{2}\left(\omega^{2}-1\right)}{\beta e}$.

If $\omega^{2}=1, a_{s}=2-\frac{\beta e}{3 h} \sin 2 \theta_{s}, \cos 2 \theta_{s}=\frac{-4 \beta}{e}-\frac{3 h^{2}}{8 \beta e}$ on condition that $8 \beta e \geq$ $32 \beta^{2}+3 h^{2}$.

\section{Stability conditions}

The stability study is based on the equations of variation 


$$
\begin{aligned}
& (\delta a)^{\bullet}=\left(\varepsilon \frac{\partial A_{1}}{\partial a}+\varepsilon^{2} \frac{\partial A_{2}}{\partial a}\right) \delta a+\varepsilon^{2} \frac{\partial A_{2}}{\partial \theta} \cdot \delta \theta, \\
& (\delta \theta)^{\bullet}=\varepsilon^{2} \frac{\partial B_{2}}{\partial a} \delta a+\varepsilon^{2} \frac{\partial B_{2}}{\partial \theta} \delta \theta
\end{aligned}
$$

from which the characteristic equation can be established

$$
\lambda^{2}-\left\{\varepsilon \frac{\partial A_{1}}{\partial a}+\varepsilon^{2}\left(\frac{\partial A_{2}}{\partial a}+\frac{\partial B_{2}}{\partial \theta}\right)\right\} \lambda+\left\{\varepsilon^{3} \frac{\partial A_{1}}{\partial a} \frac{\partial B_{2}}{\partial \theta}+\varepsilon^{4} \ldots\right\}=0 .
$$

The two sufficient stability conditions are:

$$
\begin{aligned}
& \varepsilon \frac{\partial A_{1}}{\partial a}+\varepsilon^{2}\left(\frac{\partial A_{2}}{\partial a}+\frac{\partial B_{2}}{\partial \theta}\right)<0, \\
& \varepsilon^{3} \frac{\partial A_{1}}{\partial a} \cdot \frac{\partial B_{2}}{\partial \theta}+\varepsilon^{4} \ldots \cdots>0 .
\end{aligned}
$$

With regard that $a_{s}=a_{*}+\varepsilon a_{1}$ the condition (6.2) can be written as

$$
\varepsilon \frac{\partial A_{1}\left(a_{*}\right)}{\partial a}+\varepsilon^{2}\left\{a_{1} \frac{\partial^{2} A_{1}\left(a_{*}\right)}{\partial a^{2}}+\frac{\partial A_{2}\left(a_{*}\right)}{\partial a}+\frac{\partial B_{2}\left(a_{*}, \theta_{s}\right)}{\partial \theta}\right\}<0
$$

or, in pratice (by neglecting the terms of order $\varepsilon^{2}$ )

$$
\frac{\partial A_{1}\left(a_{*}\right)}{\partial a}<0
$$

Since $A_{1}=h a\left(1-\frac{a^{2}}{4}\right)$ and $a_{*}=2$ we have $\frac{\partial A_{1}\left(a_{*}\right)}{\partial a}=-h<0$ so that the first condition of stability is always satisfied.

By neglecting the terms of order $\varepsilon^{4}$, the second stability condition can be simplified as

$$
\begin{aligned}
\frac{\partial A_{1}\left(a_{*}\right)}{\partial a} \cdot \frac{\partial B_{2}\left(a_{*}, \theta_{s}\right)}{\partial \theta} & =-h \frac{\partial B_{2}\left(a_{*}, \theta_{s}\right)}{\partial \theta}>0 \\
\text { i.e. } \quad \frac{\partial B_{2}\left(a_{*}, \theta_{s}\right)}{\partial \theta} & <0 .
\end{aligned}
$$

As an illustration let us form the stability conditions of the system examined in $\S 2$ (the case of external excitation in subharmonic resonance of order $\frac{1}{3}$ ). We have

$$
\frac{\partial B_{2}}{\partial \theta}=\frac{-3 h e a \cos 3 \theta}{64 \omega^{2}}, \quad \frac{\partial B_{2}\left(a_{*}, \theta_{s}\right)}{\partial \theta}=\frac{-3 h e \cos 3 \theta_{s}}{32 \omega^{2}}=\frac{-3 h a_{1}}{2 \omega^{2}} .
$$


The stability condition (6.7) is satisfied with $a_{1}>0$ and does not satisfied with $a_{1}<0$. This means that synchronized oscillations corresponding to a larger amplitude $\left(a_{s}=2+\varepsilon a_{1}>2\right)$ are stable; those corresponding to a smaller amplitude $\left(a_{s}=2-\varepsilon a_{1}<2\right)$ are unstable.

\section{Conclusion}

The examples examined above show that there exist self-excited systems for which the synchronization occurs only in the second approximation. The asymptotic method can successfully be used to study these systems; the stability conditions can easily be established.

This publication is completed with the financial support from The Council for Natural Science of Vietnam.

\section{REFERENCES}

1. Bogoliubov N. N., Mitropolskii Yu. A. Asymptotic methods in the theory of nonlinear oscillations, Moscow, 1974.

2. Malkin J. G. Some problems in the theory of nonlinear oscillations, Gostekhizdat, Moscow, 1956.

3. Mitropolskii Yu. A., Nguyen Van Dao. Applied asymptotic methods in nonlinear oscillations, Kluwer Academic Publishers, 1996.

4. Nguyen Van Dao, Nguyen Van Dinh. Interaction between nonlinear oscillating systems. Vietnam National University Publishing house, Hanoi, 1999.

Received December 10, 2002

\section{HIỆN TUỢNG ĐỒNG Bộ ở XẤP Xİ THỨ HAI}

Xét hiện tượng đồng bộ ở một số hệ tự chấn chịu kích động cưỡng bức hoặc thông số. Đặc điểm các hệ này là ở xấp xỉ thứ nhất có chế độ tự chấn dừng ổn định và chế độ này được đồng bộ hóa ở xấp xỉ thứ hai. 